\title{
TRASNFERENCIA Y EMPATÍA ${ }^{1}$
}

\section{TRANSFERENCE AND EMPATHY}

\author{
Pablo Gelsi \\ Universidad Católica del Uruguay, Uruguay
}

\begin{abstract}
Resumen: El autor reflexiona sobre la posible interrelación entre los conceptos de "Transferencia" tal como lo concibe C.G.Jung y la Psicología Analítica y el de "Empatía”en la Psicología Centrada en el Cliente de Carl Rogers, especialmente en su aplicación en procesos psicoterapéuticos.

La relación psicoterapéutica puede incluir elementos de estas dos perspectivas, que pueden ser consideradas no como opuestas, sino más bien como complementarias. El énfasis en la consideración de los aspectos inconscientes de dicha relación implica necesaria y concomitantemente de un contexto donde la comprensión empática esté operando.

Para ilustrar esta posible confluencia de los aportes de ambas corrientes se señalan tres actitudes básicas del psicoterapeuta frente al fenómeno de la Transferencia del lado de la Psicología Analítica y las actitudes básicas del Psicoterapeuta según Rogers.
\end{abstract}

Palabras clave: transferencia, empatía, psicoterapia, uno mismo, sí mismo, individuación.

\begin{abstract}
The author offers a reflection around the possible interrelationship between the concepts of "Transference"”, as conceived by C.G.Jung and Analytical Psychology and that of "Empathy", coming from Client Centered Therapy of Carl Rogers, especially in their application in psychotherapeutic processes. The psychotherapeutic relationship can include elements from these two perspectives, that can be considered no as opposed, but as complementary. The emphasis in the consideration of the unconscious aspects of this relationship implies necessarily and concomitantly a context in which emphatic understanding is operating.

In order to illustrate this possible confluence of both approaches, three basic attitudes of the psychotherapist with regards to Transference as conceived by Analytical Psychology are stressed, alongside the three basic attitudes that should be present in the therapist according to Rogers.
\end{abstract}

Keywords: transference, empathy, psychotherapy, onself, self, individuation.

El interés que subyace a las reflexiones de este trabajo, acerca de la Transferencia y la Empatía es intentar observar algunos aspectos del fenómeno amplísimo que es la psicoterapia, a la luz de ciertos aportes de Carl Jung y de Carl Rogers.

En apariencia ambos autores no tienen mucho que ver entre sí, sin embargo a través de la experiencia en mi práctica clínica, he llegado a la conclusión de que ambos pueden complementarse. Desearía estas reflexiones significasen un aporte a este Congreso que versa justamente sobre la Multiplicidad.

El pensamiento de Carl Gustav Jung ha sido decisivo en mi labor profesional desde que en $\mathrm{mi}$ juventud, cuando mi director espiritual de enton- ces, un sacerdote jesuita, puso en mis manos "El Secreto de la Flor de Oro" (Jung 1972).

Desde entonces y hasta el presente, con la reciente aparición del "Libro Rojo" (Jung 2009) sus ideas siguen despertando mi admiración y mi agradecimiento a este maestro que con tanto coraje nos ha guiado por caminos que eran entonces desconocidos y que ahora transitamos con más seguridad que la que le tocó a él.

Leí a Carl Rogers cuando estudié Psicología en Estados Unidos, en la década de los años 70, etapa en la que y la corriente psicoterapéutica que fundara, la "Psicoterapia Centrada en el Cliente" formaban parte de la corriente llamada "Psicología Humanística". 
Una corriente poco estimada entonces, porque era la época en la que estaban en pleno apogeo Skinner y Watson, el Comportamiento Animal y la Psicología Fisiológica entre otros temas de interés para la investigación en Psicología, y sobre todo la Psicología Experimental.

La noción de salud mental para Jung y para Rogers está ligada a la dinámica de un proceso natural en el desarrollo humano. Se alcanza cuando funcionan en una cierta armonía todos los factores psicológicos que conforman a la persona humana.

En contrapartida la enfermedad está ligada al estancamiento de ese proceso, a la unilateralidad y desconocimiento de la completud de la personalidad.

El objetivo de la psicoterapia para Jung es alcanzar la Individuación y el entrar en contacto con la verdad y el saber infinitos del mundo arquetípico. Para Rogers es más bien el deseo de ser uno mismo, aunque esa mismidad no comprende la noción de inconsciente.

La noción de empatía que Rogers propugna, conjuga o permite asimilar la propuesta de neutralidad del terapeuta que apoyaba Freud, la búsqueda de la individuación de Jung y el alcanzar la alteridad que propone Byington.

Creo que hemos tenido la fortuna de haber contado con maestros que resistieron la posibilidad de sugestionar y de tornar dependientes a otros, de buscar la posibilidad de que cada persona intente encontrarse a sí misma y de reconocer que todo eso se logra junto con otros.

Creo también que la noción de transferencia de la tradición psicodinámica, especialmente la de la Psicología Analítica puede complementar la comprensión de la relación terapéutica tal como la concibe Rogers.

Marco Antonio Recuero (Recuero: 2008), que presenta un excelente paralelo entre la vida y la obra de ambos autores, sostiene que ellos nunca se conocieron. Es probable que Jung nunca leyera a Rogers y este último apenas menciona la obra de Jung.

Si nos basáramos en los Tipos Psicológicos él sería un Introvertido con predominio de la función Intuición y Rogers un Extravertido (Jung: 1925) que describe Jung, con predominio de la función Sentimiento.

Ortega y Gasset (Ortega y Gasset: 1984), el filósofo español, sostenía que la Humanidad es un concepto vasto y generoso, aludiendo a los tipos de hombres tan dispares que se pueden alojar en ella sin estorbarse.
Lo que aquí nos interesa es que cada tipo de hombre observa la realidad del mundo desde su propia perspectiva, que es única, pero a la vez, y queremos creerlo, compartible y sobre todo, complementaria con la de otros.

Mi propósito final es intentar mostrar la articulación de ambos enfoques, observar ciertos fenómenos que ocurren en los procesos psicoterapéuticos, que deben reunir los aportes de ambos para ser exitosa.

El proceso terapéutico es para ambos autores un tipo de relación dialéctica, esto es un diálogo entre dos personas (el psicoterapeuta y el paciente) que resultan transformadas por el proceso mismo de la interacción. Resaltan la importancia del vínculo en el éxito de la psicoterapia. No es sólo la búsqueda de la salud mental sino más bien de un cambio en la relación con uno mismo, con otros y con el mundo.

\section{LA RELACIÓN DE TRANSFERENCIA EN LA VISIÓN PSICODINÁMICA Y SU PRESENCIA EN TODA RELACIÓN HUMANA}

La noción de Transferencia revolucionó el mundo de la psicoterapia, porque introdujo la idea de que existían sentimientos y emociones, deseos y representaciones, cuyo origen estaba ligado a la existencia del inconciente. Tanto fue así que durante mucho tiempo fue un término reservado exclusivamente a fenómenos que ocurrían a lo largo del proceso de análisis. Pero modernamente se ha podido verificar que en realidad, dado que se trata de fenómenos de proyección es que también se presentan en toda relación humana.

Siempre me impresionó la afirmación que hace Jung en sus Memorias (Jung y Jaffé, 1989, p. 357) de que no tenía paciencia con la gente (aunque agrega, "excepto con mis pacientes") "Para algunas personas estaba continuamente presente y cercano a ellas, mientras estuviesen relacionadas con mi mundo interno; pero entonces podía suceder que yo no estuviese más con ellas, porque ya no quedaba nada que me ligara a ellas. Tuve que aprender penosamente que las personas seguían existiendo aún cuando no tuvieran nada para decirme."

Me parece que el interés de Jung por el mundo de los arquetipos influyó notablemente sobre la concepción de lo que consistía el análisis junguiano y su práctica, inevitablemente volcado al encuentro con el inconsciente colectivo y los símbolos arquetípicos. 
Algunos autores, como Mario Jacob (Jacoby, 1984), intentaron desde el inicio rescatar los aspectos personales y relacionales del análisis junguiano y hoy día no se discute que esta dimensión de encuentro entre personas es básica en el análisis.

Lo que sugiero es que lo que fue natural desde el inicio en Rogers, el encuentro interpersonal, la terapia centrada en el cliente, se fue incorporando luego de que se revalorizara la dimensión personal del paciente y del terapeuta.

Cuando Sigmund Freud descubrió el fenómeno de la transferencia, no sé si intuyó el tremendo significado que tendría para comprender toda relación humana. Sabemos sí de la importancia que le concedía en la relación analítica, al punto de coincidir con Jung en aquella famosa conversación que duró 13 horas en que la transferencia era "el alfa y el omega de todo tratamiento psicoanalítico" (Jung CW 16, p.358).

Para 1907 vendrá su descubrimiento de las contratransferencia, que Freud consideró un efecto en el analista de la transferencia de sus pacientes. Recién en 1949 Paula Heimann resaltó la contribución de la realidad psíquica del analista a la aparición del fenómeno a la relación, así como su valor diagnóstico.

Pero Freud llegó a proponer, o así se lo entendió, que el analista realizara el mayor sacrificio que se le puede pedir a un curador, a un ayudador, o sea, no existir como persona y transformarse en un espejo de las proyecciones de sus pacientes. La Transferencia en definitiva es proyección, sólo que es vivida por el paciente en el aquí y en el ahora. De allí viene su carga energética y sus posibilidades de brindar la cura al paciente. En lugar de repetir, hay que hacer consciente el pasado y elaborar e integrar los complejos infantiles para evitar la condena de un eterno sufrimiento.

Creo que con Jung, el tema de la transferencia y la relación humana se volvió aún más complejo. Es que teoriza el tema de la transferencia arquetípica, especialmente en su obra "Psicología y Transferencia" (Jung: CW16) la que muestra cómo la relación humana es el símbolo de la unión mística entre el inconsciente y la conciencia, el símbolo por excelencia de la "coniunctio oppositorum", el opus alquémico y el logro de la totalidad.

Los aportes de Groesbeck (Groesbeck: 1975), de Jacobi (Jacoby: 1984), de Guggenbuhl-Craig (Gugenbuhl-Craig:1971), humanizan este concepto de la transferencia arquetípica, mostrándonos que todos los seres humanos, que por serlo, padecemos de una herida incurable, no debemos proyectar la curación, la salvación en el otro y buscar en cambio la curación posible en nosotros mismos.

La transferencia, sea personal o arquetípica ocurre en cualquier relación entre personas, porque en su base está el fenómeno de la proyección. Cuando ocurre en la relación terapéutica llamamos a este fenómeno transferencia. Lo que ocurre allí es una alerta a lo que puede suceder en cualquier otra relación que llamamos, algo ingenuamente, de humana.

Marca la aparición de un fenómeno de despersonalización de la relación entre ambos participantes, un momento en el que ambos dejan de estar allí y dejan de ser ellos mismos. Son transportados al mundo feerico del inconsciente y se convierten en los actores representando lo que Mannoni (Mannoni, 1973) llamó "la otra escena".

Jung lo considera como la ilustración acerca de cómo los complejos "nos tienen a nosotros". La demostración más dramática de todo esto a mi juicio está en la transferencia erótica, en la que el amado es en realidad la línea de defensa más profunda contra el progreso del proceso analítico.

Jung consideraba que cualquier análisis en profundidad y de larga duración, difícilmente se libraba de este fenómeno de la transferencia. En algún escrito la considera un obstáculo (Jung: CW 18, parr. 678). Prefería con todo, que fuese más del tipo de la transferencia positiva, de curso más manso y que facilita la relación de trabajo entre ambos participantes.

\section{LAS TRES ACTITUDES BÁSICAS DEL PSI- COTERAPEUTA FRENTE AL FENÓMENO DE LA TRANSFERENCIA}

Es de vital importancia cuál será la actitud del terapeuta en relación a la aparición del fenómeno transferencial. La primera actitud que debe activarse en el terapéutica es la de sostener, por así decirlo, las proyecciones del paciente, resistirlas en el sentido de ser capaz de soportarlas, no rechazarlas. Debe ser capaz de darse cuenta de las reacciones contratransferenciales que esta situación le genera y nunca actuarlas.

Debe ser capaz de aceptar que la relación con sus paciente está pasando por una fase "no 
humana" para el paciente. El no está allí para él. Quizás esté en el lugar de un progenitor, quizás sea un personaje mitológico o alguien salido de un cuento de hadas. Pero él mismo se ha ido, se ha prestado a este serísimo juego donde han sido llamadas del pasado personal y del pasado de la especie, las figuras esenciales para el misterioso drama que es la curación.

En segundo lugar, el terapeuta debe darse cuenta qué papel se le pide que juegue en ese drama. Normalmente este papel es el del curador. Si en determinado momento él no representa la esperanza de la cura, el proceso terapéutico no podrá avanzar.

Nosotros que sabemos que en realidad son los dioses los que curan, debemos aceptar cargar, en nuestra fragilidad de humanos esta terrible y fascinante esperanza. Resonaría aquí la tentación eterna de la serpiente en el Jardín del Eden: "Sereis como dioses".

En tercer lugar, el terapeuta debe ofrecerse como mediador. Primero es no rechazar la proyección, segundo es cargarla y tercero mediar. Mi impresión es que no nos hemos detenido demasiado sobre esta función que tiene en Hermes como su representante en la mitología griega.

Son varios las características que el mitólogo brasilero Junito Brandao (Brandao: 2002) atribuye a Hermes. Me referiré a unos pocos que hacen al presente trabajo. Es según le dice Zeus es el compañero del hombre y su escucha. Es protector de los viajantes y de los caminos, el intérprete de la voluntad de los dioses y mensajero de éstos ante los hombres. Es el dios del comercio y de la negociación, es astuto e inventivo, es el trasmisor de la ciencia secreta.

Creo que tenemos que pensar al terapeuta como representante de Hermes, en la transferencia arquetípica, debido en primer lugar aceptarlo como su protector en el viaje que emprende con su paciente y como conocedor del camino que emprenderán.

En segundo lugar, aceptarlo como aquel factor que puede entonces actuar como mediador entre el Sí mismo y el Yo, por su afinidad con la conciencia y el contacto con la divinidad suprema.

Tercero, por su carácter de negociador. Es sabido que para que una negociación resulte exitosa, es necesario que todas las partes renuncien a algo. El terapeuta navega entre los lugares oscuros y los luminosos de sus pacientes para intentar el logro de una síntesis armoniosa y siempre precaria, de su conducta y de su personalidad.

En cuarto lugar, por sus astucia y su inventiva. Vez tras vez Jung nos advierte que cada proceso terapéutico es peculiar y único y por lo tanto enfrentar a cada paciente es empezar de nuevo.

Por último y sin proponérselo, el terapeuta es el trasmisor para el paciente del conocimiento que posee en sí mismo, aunque no lo sepa. Es el que sabe y cuida de que el propio curador del paciente tenga finalmente posibilidades de manifestarse. Un conocimiento que trasciende a ambos y que es manifestación en lo personal, del todo el saber que nuestra especie ha acumulado.

\section{LAS ACTITUDES BÁSICAS DEL TERAPEUTA SEGÚN ROGERS}

De acuerdo a lo que afirma Rogers (Rogers: 1961), ya la investigación en psicoterapia en su tiempo, demuestra que se deben dar al menos tres condiciones o actitudes básicas en el terapeuta para el logro de un proceso exitoso.

Llama a la primera Congruencia o integridad. Para él ser auténtico es ser verdaderamente lo que se es como primer paso para lograr que el paciente experimente y sienta lo que él mismo es, lo que como vimos anteriormente, es la meta de la terapia centrada en el cliente.

El terapeuta deberá poder reflejarle al paciente sus propios sentimientos y vivencias en relación a él, aun si se tratare de elementos por así decirlo, negativos, porque es una manifestación de su persona real y por lo tanto imperfecta.

La segunda es lo que denomina la Aceptación Positiva Incondicional. Parte de la base de que el terapeuta debe aceptarse a sí mismo de ese modo, con la finalidad de conocer sus fallas y sus talentos, para poder hacerlo con el paciente. Sostiene que es el modo cómo las relaciones interpersonales se vuelven reales, en lugar de ser el resultado de nuestros deseos y necesidades.

Para él es una fuente de gran satisfacción el poder aceptar plenamente a otra persona, y encuentra que cada vez que lo logra se siente cada vez menos llamado a arreglar las cosas que le pasan al otro. Puede escucharlo mejor, explorar con él las alternativas y las soluciones para los verdaderos problemas que trae. 
Se trata de una actitud sumamente difícil pero muy deseable, que significa que el terapeuta no quiere que la persona sea como él desea que sea, sino que él mismo busque ser lo que es realmente.

La tercer actitud o condición es la Comprensión Empática, o simplemente la Empatía, un concepto sobre el que escribió mucho y enfatizó mucho como uno de los factores claves para el éxito de la psicoterapia.

Se trata de la comprensión íntima del otro, del mundo interno del otro, tal como éste lo vive, aunque no sepa con claridad cómo lo vive. Es la percepción del otro en uno, como alguien que no soy yo, pero que me resulta tan familiar como lo soy para mí mismo.

Es una vivencia paradojal, porque a diferencia de la transferencia, no es una proyección (y por lo tanto inconsciente) sino la vivencia de otro como otro en mí. No es una identificación sino la impresión de la vivencia del otro como si fuera mía, con la posibilidad de discriminarme de ella y continuar siendo yo mismo y él, él, separados y juntos a la vez.

Rogers ofrece por supuesto otras pistas, otras conclusiones sobre lo que es esencial en definitiva para que el terapeuta consiga realizar la tarea, pero que sería largo de enumerar.

Sólo les cuento algunas pocas, que a mi entender guardan una directa relación con cosas sobre las que Jung concluyó respecto de la práctica de la psicoterapia.

Valoró su propia experiencia, no dejó que la evaluación por parte de otros fuese una guía para él, el haber podido encontrar significado y orden en la experiencia, el que lo que es vivido como muy personal es muchas veces una ley universal, el que las personas se mueven constructiva y positivamente. Por último, la idea de que "la vida es un proceso cambiante, que fluye, un proceso en el cual nada resulta fijo" (Rogers: 1961, p. 27).

Creo que todos podemos estar de acuerdo con Rogers que sin esas tres condiciones o actitudes el proceso psicoterapéutico está destinado al fracaso, como lo demuestra la investigación sobre los resultados de las distintas terapias.

Rogers no le dio demasiada importancia al tipo de técnica que se usaran e indirectamente al tipo de orientación o marco teórico. Concuerda con Yoram Kaufmann en que "no hay una técnica única que sirva a todos los analizandos" (Kaufmann 2009, p.17) Pero propuso que estas actitudes fueran un sine qua non para todo tipo de abordaje terapéutico.

Nosotros los junguianos nos preguntamos qué lugar le da al inconsciente, o a la percepción del inconsciente, al lugar del terapeuta como mediador entre la conciencia y el inconsciente. En principio Rogers no se ocupa del tema.

De un libro editado después de su muerte, pero que contienen dos capítulos suyos (Santos: 1987), se puede pensar que Rogers tenía en mente una cuarta actitud o condición del terapeuta, que a mi entender lo acerca a la concepción junguiana del proceso terapéutico, del concepto de la transferencia y de papel del curador.

Cuando el terapeuta está "en forma", puede descubrir que experimenta una característica suplementaria. Puede constatar que cuando está muy cerca de su centro, de su yo intuitivo, en cierta forma en contacto con una parte desconocida de él, tal vez en un estado un poco alterado de conciencia durante la relación terapéutica, entonces cualquier cosa que haga parece ser totalmente curativa.

Su sola presencia es liberadora y de completa ayuda. No existe nada que pueda hacer para forzar esa experiencia, pero cuando se puede relajar y estar cerca de su centro trascendental, puede comportarse de maneras extrañas e impulsivas en la relación, maneras que no se podrían justificar racionalmente y que no tienen nada que ver con sus procesos de pensamiento. Pero estos comportamientos extraños, de manera curiosa, resultan acciones "correctas". En estos momentos parece que el espíritu interno penetra en el espíritu interno del otro. La relación entre ambos se trasciende a sí misma y se torna parte de algo mayor. Se producen procesos de crecimiento profundos, hay un cambio en la energía y aparece la cura.

Concluyendo, quiero pensar que quizás fue su modo de decir que es en el encuentro con el Sí Mismo y con la trascendencia de los opuestos y de las aparentes contradicciones y diferencias, que la persona logra, en el proceso que es la vida, una vivencia aunque más no sea fugaz, de la unión con la totalidad del cosmos y de la empatía con toda la especie humana, que según Jung es la meta del Proceso de Individuación.

Como reflexiones finales, estimo que he brindado algunos elementos que permiten establecer la pertinencia de utilizar ambas visiones a la hora de encarar en los hechos, algunos procesos psicoterapéuticos. 
La empatía, si bien puede ser reconocida conscientemente, a mi modo de ver también implica una captación inconsciente de la otra persona. Los fenómenos inconscientes de la relación tornan por otro lado imprescindible la presencia de un yo consciente en diálogo con ellos.

De la misma manera, el tener una visión diversa de la estructura de la personalidad humana, no impide la coincidencia de los objetivos de la intervención psicoterapéutica, en definitiva, el alivio del sufrimiento psíquico, la necesidad de realizar la propia individualidad en uno mismo y con otros.

\section{REFERENCIAS}

Byington, Carlos (2008). Psicologia Simbólica Junguiana. El viaje de humanización del cosmos en busca de la iluminacíon. São Paulo: Linear.

Brandâo, J. de S. (1986). Mitologia Grega 1. Petrópolis: Editorial Vozes.

Jung, C.G. (1966). Collected Works of C.G. Jung (vol.16). Groesbeck, C.J. (1975). The Archetypal Image of the Wounded Healer. Journal of Analytical Psychology, 20 (2):122-145.
Guggenbuhl-Craig, A. (1971). Power in the Helping Professions. New York: Spring Publications.

Jacoby, M (1984). The Analytic Encounter: Transference and Human Relationship. Toronto: Inner City Books.

Jung, C. G. (1943). Tipos psicológicos. Buenos Aires: Editorial Sudamericana.

Jung, C. G. (1989). Memories, Dreams and Reflections. NewYork: Vintage Books.

Jung, C.G. (2009). The Red Book - Liber Novus. New York: W. W. Norton \& Company.

Jung, C. G. \& Wilhelm, R. (1982). El secreto de la flor de oro. Buenos Aires: Paidós.

Mannoni, O. (1973) La otra escena. Claves de lo imaginario. Buenos Aires: Amorrortu Editores.

Ortega y Gasset, J. (1994). Estudios sobre el Amor. Madrid: Ediciones del Prado.

Recuero, M. A. (2008) Los modelos terapeúticos de Carl Jung y Carl Rogers. Santiago de Chile: Ediciones Universidad Católica de Chile.

Rogers, C. (1961). On Becoming a Person: A Therapist's View of Psychotherapy. New York: Houghton Mifflin Company.

Santos A.M. \& Villas-Boas, M.C. (2004). Quando Fala o Coração: A essência da psicoterapia centrada na pessoa. São Paulo: Vetor Editora.

1 (N de la Ed). El Prof. Pablo Gelsi, es uno de los responsables del desarrollo de la Maestría en Psicología Clínica con orientación en Psicología Analítica de la Facultad de Psicología de la Universidad Católica del Uruguay; reconocido Psicoteraputa junguiano y docente a nivel de grado y postgrado en diversas Universidades, ha sido también Decano de la Facultad de Psicología. La ponencia que aquí se publica fue aceptada y presentada en el XVIII Congreso de la Asociación Internacional de Psicología Analítica, Montreal, Canadá, 2010. Revisada por el autor y arbitrada.

Para citar este artículo:

Gelsi, P. (2011). Trasferencia y Empatía. Ciencias Psicológicas V (1): 117-122. 